\title{
Communication in Health - A Challenge
}

\section{Vihang Mazumdar}

Retd. Professor and Head, Department of Community Medicine, Medical College, Baroda, Gujarat, India

Correspondence : Dr. Vihang Mazumdar, Email: vihang.mazumdar@gmail.com

The recent discussion nationally on vaccine hesitancy and reluctance to follow Covid appropriate behaviour has once again brought focus on how communication in health has remained a challenge after all these years. Why haven't we convinced mothers to wash hands and use fluids available at home for diarrhoea prevention? Why do doctors fail to give proper advice to patients even for their own prescription? Why aren't proper instructions given while dispensing IFA? Why are the four key messages not given by health workers during vaccination?

One common thread runs through all these: lack of communication.

It becomes difficult to comprehend why and how we fail considering that on one hand "unlimited talktime" is an absolute must for us and yet we are very reticent when it comes to communicating where it matters. On one hand we see the all pervasiveness of products like noodles and on the other we fail to convince mothers to give exclusive breast feeding and complementary feeding with the same success.

Over the years the jargon used, health literacy, health awareness, health education, mass media communication, advocacy, counselling, interpersonal communication, behaviour change communication to social and behaviour change communication has changed. What remains constant is the fact that unsolicited advice is not welcome.

In the triad of Message, Medium and Messenger the messages are often bland, having to conform to scientific and political correctness; the varieties of media are ever expanding with their own

\begin{tabular}{|c|l|l|}
\hline Quick Response Code & Access this article online & How to cite this article: \\
\hline & Website : & Mazumdar V. Communication in Health - A Challenge. \\
Healthline 2021; 12(1):03-04. & \\
\cline { 2 - 3 } & DoI : & \\
\hline
\end{tabular}

requirements as to the format of messages. Finally the huge impact of the messenger cannot be underestimated.

Let us for a moment dwell upon the issue of vaccine hesitancy. While everyone expected the vaccine to be welcomed with open arms, a contrary response was met with much chagrin. If the vaccine was presented after a few weeks after adequate data regarding safety and efficacy was made available in public domain. The details of vaccine been communicated by competent authority. A time window announced for availability of vaccine for health professionals. Would the response have been different? Perhaps... Sometimes a very aggressive sales pitch makes a client wary. Social media also had an immense role in vitiating the atmosphere. Inherently, people are more receptive to bad news. Any negative report, without confirming veracity or authenticity of source is lapped up with an "Aha! The crime has been exposed!" and promptly forwarded to a large number of equally gullible folks.

The messenger challenge is a difficult one to overcome. Looking at the recent experiences of MR (Measle-Rubella) and Rota vaccine campaigns, traditional models of local leaders, religious leaders, etc. did not give equal dividends in rural and urban areas. Lack of success of local leaders in getting day to day problems solved may be the reason urban population was not inspired to follow his/her appeals.

The celebrity endorsement option also at times may follow the law of diminishing returns. While in 
early days "Do boond zindagi ke" struck a chord, messages during later campaigns did not get the same response as they got lost among many messages by the same person selling hair oil, gold loans, jewellery and many more products. I would be as likely to get my child vaccinated in response to that message as I would be likely to buy the hair oil.

Therefore the challenge for us is to seek new avenues to engage with people such that there is credibility and higher acceptance. I feel, especially for urban areas, that the extinction of the once common species of "MBBS family doctor" has created a void as a link between the health authorities and people. As a guide, philosopher and friend who knew and understood the needs of the family, he/she was ideally placed as a reliable and credible source to endorse something.

Once upon a time (and even now) KAP (Knowledge, Attitude and Practice) studies were the bread and butter of every resident attending a conference. Every postgraduate has written a very elaborate plan to create awareness (and even perhaps eliminate) a myriad of health issues in their examination papers and assignments, now the time has come to put them to test, to explore new ideas to create awareness regarding health issues and create the impact they deserve. 